\title{
Answers to paediatric crossword puzzle 35
}

Manouri P Senanayake ${ }^{1,2}$, A S Athapathu ${ }^{2}$

Sri Lanka Journal of Child Health, 2017; 46(3): 296

DOI: http://dx.doi.org/10.4038/sljch.v46i3.8340

\begin{tabular}{|c|c|c|c|c|c|c|c|c|c|c|c|c|c|}
\hline${ }^{1} \mathrm{~W}$ & $\mathrm{I}$ & $\mathrm{N}$ & $\mathrm{K}$ & $\mathrm{I}$ & $\mathrm{N}$ & ${ }^{2} \mathrm{G}$ & & & & & & ${ }^{3} \mathrm{I}$ & \\
\hline $\mathrm{A}$ & & & & & & $\mathrm{R}$ & & ${ }^{4} \mathrm{~V}$ & ${ }^{5} \mathrm{E}$ & $\mathrm{G}$ & $\mathrm{A}$ & $\mathrm{N}$ & $\mathrm{S}$ \\
\hline $\mathrm{R}$ & & & ${ }^{5} \mathrm{~S}$ & $7 \mathrm{~L}$ & $\mathrm{E}$ & $\mathrm{E}$ & $\mathrm{P}$ & & $\mathrm{M}$ & & & $\mathrm{A}$ & \\
\hline${ }^{8} \mathrm{~T}$ & ${ }^{9} \mathrm{O}$ & $\mathrm{F}$ & & $\mathrm{I}$ & & $\mathrm{E}$ & & & $\mathrm{O}$ & & & $\mathrm{H}$ & \\
\hline & $\mathrm{X}$ & & ${ }^{10} \mathrm{~T}$ & $\mathrm{~V}$ & & $\mathrm{~N}$ & & & ${ }^{11} \mathrm{~T}$ & $\mathrm{~S}$ & $\mathrm{H}$ & & ${ }^{12} \mathrm{~B}$ \\
\hline${ }^{13} \mathrm{X}$ & $\mathrm{Y}$ & & & $\mathrm{E}$ & & & ${ }^{14} \mathrm{C}$ & & $\mathrm{I}$ & & & & $\mathrm{A}$ \\
\hline & ${ }^{15} \mathrm{M}$ & $\mathrm{I}$ & $\mathrm{T}$ & $\mathrm{R}$ & ${ }^{16} \mathrm{~A}$ & $\mathrm{~F}$ & $\mathrm{~A}$ & $\mathrm{~N}$ & $\mathrm{O}$ & $\mathrm{F}$ & $\mathrm{F}$ & & $\mathrm{C}$ \\
\hline & $\mathrm{E}$ & & & & $\mathrm{S}$ & & $\mathrm{S}$ & & $\mathrm{N}$ & & & & $\mathrm{T}$ \\
\hline & ${ }^{17} \mathrm{~T}$ & $\mathrm{U}$ & ${ }^{18} \mathrm{G}$ & & $\mathrm{D}$ & & $\mathrm{T}$ & & ${ }^{19} \mathrm{~A}$ & $\mathrm{~L}$ & ${ }^{20} \mathrm{I}$ & $\mathrm{C}$ & $\mathrm{E}$ \\
\hline & $\mathrm{R}$ & & $\mathrm{L}$ & & & & ${ }^{21} \mathrm{~S}$ & $\mathrm{I}$ & $\mathrm{L}$ & & $\mathrm{N}$ & & $\mathrm{R}$ \\
\hline${ }^{22} \mathrm{M}$ & $\mathrm{Y}$ & $\mathrm{X}$ & $\mathrm{O}$ & $\mathrm{M}$ & ${ }^{23} \mathrm{~A}$ & & & & & & $\mathrm{U}$ & & $\mathrm{I}$ \\
\hline $\mathrm{P}$ & & & $\mathrm{V}$ & & $\mathrm{D}$ & & & & ${ }^{24} \mathrm{M}$ & $\mathrm{I}$ & $\mathrm{L}$ & $\mathrm{I}$ & $\mathrm{A}$ \\
\hline${ }^{25} \mathrm{~A}$ & $\mathrm{P}$ & $\mathrm{P}$ & $\mathrm{E}$ & $\mathrm{N}$ & $\mathrm{D}$ & $\mathrm{I}$ & $\mathrm{X}$ & & $\mathrm{R}$ & & $\mathrm{I}$ & & \\
\hline & & & $\mathrm{S}$ & & & & & ${ }^{26} \mathrm{~K}$ & $\mathrm{I}$ & $\mathrm{D}$ & $\mathrm{N}$ & $\mathrm{E}$ & $\mathrm{Y}$ \\
\hline
\end{tabular}

${ }^{1}$ Department of Paediatrics, Faculty of Medicine, Colombo, ${ }^{2}$ Lady Ridgeway Hospital for Children, Colombo 